DOI: 10.20472/IAC.2018.044.042

\author{
UTTAM KUMAR SARKAR \\ INDIAN INSTITUTE OF MANAGEMENT CALCUTTA, India
}

\title{
INFERENCE UNDER A NEW EXPONENTIAL-EXPONENTIAL LOSS CAPTURING SPECIFIED PENALTIES FOR OVER- AND UNDER-ESTIMATION
}

\begin{abstract}
:
Asymmetric loss functions have gained enormous importance over the years, with particular relevance to situations where over- and under-estimation of the parameter of interest are considered not of equal consequence. In particular, the linear-exponential (LINEX) loss has been studied and used quite extensively in classical and Bayesian inference. While LINEX loss nicely captures whether over- or under-estimation has a more serious impact, it falls short of incorporating any prior knowledge about the relative penalty for over- vis-à-vis that for under-estimation. Thus, if such prior knowledge is available as happens in many practical situations, notably in finance, medicine and reliability theory, among others, then there is a pressing need for devising a loss function that accounts for this information and hence is more realistic than the LINEX loss.

More specifically, suppose the ground realities in a given situation demand that over-estimation needs to be penalized k times the penalty of under-estimation, where k is known. Clearly, over-estimation gets more penalized than under-estimation if $k>1$ and it is the other way round if $k$ $<1$. Naturally, taking care of the explicit knowledge of $k$ will entail more sensible statistical inference. The present article incorporates this information via a novel exponential-exponential loss function, named EXEX loss, designed using the sigmoid function. Unlike the linear-exponential LINEX loss which behaves more like exponential for over-estimation and rather linear for under-estimation, the proposed EXEX loss function is exponential under both kinds of estimation errors. We show how, in the inference process, this allows the experimenter to directly incorporate knowledge of the relative penalty as mentioned above via the use of the EXEX loss function, with its characteristics appropriately tuned. The ideas are demonstrated by showing how the EXEX loss works in the estimation of a normal mean and related problems. We also compare and contrast the performances of LINEX and EXEX losses in terms of risk.
\end{abstract}

\section{Keywords:}

Estimation, Squared error loss, Asymmetric loss

JEL Classification: C18, C13, C00 